\title{
Handelsgesetze als Quelle des bürgerlichen Rechtes
}

Ein Beitrag zur Lehre von der Analogie

Von

\author{
Dr. Oskar Pisko
}

o. ö. Professor an der Universităt Wien

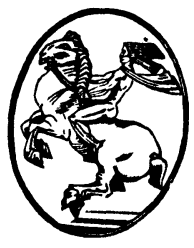

Wien

Verlag von Julius Springer

1935 
ISBN-13: 978-3-7091-9595-6

e-ISBN-13: 978-3-7091-9842-1

DOI: $10.1007 / 978-3-7091-9842-1$

Alle Rechte, insbesondere das der Utbersetzung in fremde Sprachen, vorbehalten 


\section{Vorwort.}

Nicht die bekannte und viel besprochene Erscheinung, daß die Gesetzgebung Rechtssätze, die zuerst bloß für den Handel gegolten haben, später auf den allgemeinen Verkehr ausdehnt, bildet den Gegenstand dieser Schrift. Sie will vielmehr zeigen, daß Vorschriften des Handelsrechtes, auch wenn sie nicht durch ein Gesetz zu Normen des allgemeinen bürgerlichen Rechtes erhoben worden sind, als Quellen dieses wirken können. Eine solche Wirkung eignet nämlich dem Handelsrechte sehr häufig schon auf Grund der Regeln über die Analogie. Von der Möglichkeit, das Handelsrecht auch außerhalb seines sachlichen Geltungsbereiches analog anzuwenden, hat freilich Rechtsprechung und Rechtslehre bisher nur einen recht geringen Gebrauch gemacht. Das ist auf ein eingealtertes - wenn auch nicht immer offen ausgesprochenes - Vorurteil zurückzuführen, dem die erwähnte Analogie oft begegnet. Der Versuch, ihre grundsätzliche Zulässigkeit darzutun und ihre Voraussetzungen klarzustellen, bildet den Inhalt des „,Allgemeinen Teiles“. Die dort erörterten Fragen berühren zwei Probleme: das des Lückenbegriffes und das des Umkehrschlusses. Das erstgenannte Problem hat durch Zitelmanns grundlegende $\mathrm{Ab}$ handlung „Lücken im Recht“ (1903) und durch das spätere einschlägige Schrifttum eine nahezu restlose Lösung erfahren. Ich brauchte da nur gesicherte Ergebnisse der Rechtswissenschaft zusamenzufassen und konnte auf ihnen weiterbauen. Ein ganz anderes Bild bietet die Lehre vom Umkehrschluß: Schon seine Eignung zur Rechtsgewinnung ist stark umstritten, seine gedankliche Grundlage wird nicht immer richtig erkannt und sein Verhältnis zur Analogie oft unzutreffend beurteilt. Der aufgezeigte Stand der Lehre mag den etwas breiten Raum rechtfertigen, den in dieser Schrift die - mit ihrem Gegenstande in keinem unmittelbaren Zusammenhange stehenden - Ausführungen über den Umkehrschluß einnehmen. - Die Grundsätze, zu denen ich im allgemeinen Teile gelangt bin, habe ich in dem „Besonderen Teile“ für das österreichische Recht verwertet. Eine Vollständigkeit habe ich bei der Besprechung der einzelnen Vorschriften des österreichischen Handelsgesetzbuches, die eine analoge Anwendung auf Nichtkaufleute und Nichthandelsgeschäfte gestatten, nicht angestrebt, noch weniger bei Hervorhebung der Vorschriften, die eine solche analoge Erweiterung nicht zulassen. Es war 
mir hauptsächlich darum zu tun, die Methode anzuzeigen, nach der auf dem Boden des österreichischen Rechtes darüber zu entscheiden ist, ob eine handelsrechtliche Vorschrift analog auf Tatbestände angewendet werden kann, die nicht dem Handel angehören.

Die vorliegende Studie war als ein Beitrag für die Festgabe gedacht, die die juristische Fakultät der Universität Basel im Jahre 1934 Herrn Professor Karl Wirland zu seinem siebenzigsten Geburtstag überreicht hat. Der begonnene „Beitrag“ ist jedoch im Laufe der Arbeit zu einem selbständigen Büchlein angewachsen. Es soll ein verspäteter Festgruß sein, der dem großen Meister des Handelsrechtes von einem treuen Verehrer gebracht wird.

Wien, im September 1935.

Oskar Pisko. 


\section{Inhaltsverzeichnis.}

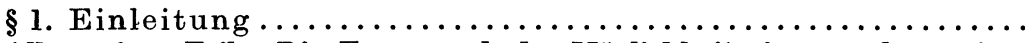

Allgemeiner Teil: Die Frage nach der Möglichkeit einer analogen Anwendung des Handelsrechtes auf handelsfremde Tatbestände. Die Voraussetzungen einer solchen Analogie...................

Erster Abschnitt: Grundsätzliche Eignung handelsrechtlicher Vorschriften zur analogen Anwendung außerhalb des Handelsrechtes. Besondere Umstände, die eine solche Eignung aus-

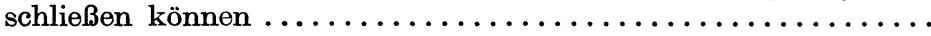

Erstes Kapitel: § 2. Die angebliche Unzulässigkeit einer analogen Ausdehnung von Ausnahmsvorschriften und von Normen des

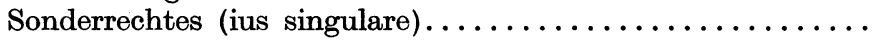
Zweites Kapitel: § 3. Der Umkehrschluß aus Handelsgesetzen als Hindernis der Analogie .................. 12

Drittes Kapitel: §4. „,Starre“ Normen des Handelsrechtes... 23

Zweiter Abschnitt: Die Voraussetzungen einer analogen Anwendung des Handelsrechtes auf Nichthandelssachen im einzelnen Falle

Erstes Kapitel : §5. Die Rechtsähnlichkeit der Tatbestände: Die Unerheblichkeit des Tatbestandselementes der Kaufmannsoder Handelsgeschäftseigenschaft ................

Zweites Kapitel: § 6. Eine Lücke des bürgerlichen Rechtes...

Besonderer Teil: Die analoge Anwendung des österreichischen Handelsgesetzbuches auf Nichtkaufleute und Nichthandelsgeschäite..$\ldots \ldots$.

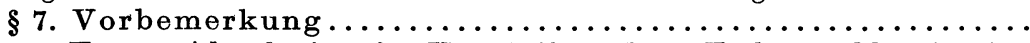

Erster Abschnitt: § 8. Vorschriften, deren Tatbestand bereits eine analoge Anwendung auf Nichtkaufleute und Nichthandelsgeschäfte

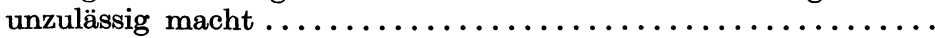

Zweiter Abschnitt: Die übrigen Vorschriften des Handelsgesetz-

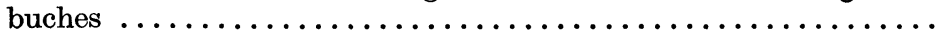

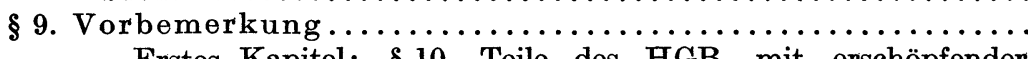

Erstes Kapitel: $\S 10$. Teile des HGB. mit erschöpfender und systematisch geschlossener Regelung des behandelten

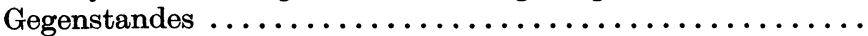
$\S$ 11. I. Abschließung und Erfüllung der Handelsgeschäfte. § 12. II. Kommissionsgeschäft, Speditionsgeschäft, Fracht-

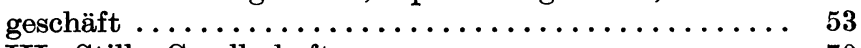

§ 13. III. Stille Gesellschaft . ............... 70

$\S 14$. IV. Offene Handelsgesellschaft und Kommanditgesellschaft $\ldots \ldots \ldots \ldots \ldots \ldots \ldots \ldots \ldots \ldots \ldots \ldots \ldots \ldots \ldots \ldots$

$\S 15$. V. Aktiengesellschaft.................. 87 
Zweites Kapitel: Teile des HGB. mit bloß bruchstückartiger Regelung des behandelten Gegenstandes ............ 94 § 16. I. Unzulässigkeit der Analogie infolge Verneinung der Rechtsähnlichkeit durch den Gesetzgeber ..........

§ 17. II. Zulässigkeit der Analogie trotz bruchstückartiger

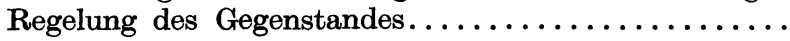
$\S$ 18. 1. Tatbestände, die sich in der Regel nur im Handelsverkehr ereignen .................

$\S 19$. 2. Beseitigung von Streit- und Zweifelsfragen und örtlichen Verschiedenheiten des bürgerlichen Rechts ......................... 99

$\S 20.3$. Rechtssätze akzessorischen Inhaltes....... 104 


\section{Abkürzungen.}

AdlCl. = Sammlung handelsrechtlicher Entscheidungen von ADLER und Clemens, fortgesetzt von FriedLändER.

ALR. = Allgemeines preußisches Landrecht.

AmtlS. = Entscheidungen des k. k. Obersten Gerichtshofes in Zivil- und Justizverwaltungssachen.

B. $\quad=$ Bundesgesetzblatt.

DBGB. = Deutsches bürgerliches Gesetzbuch.

DHGB. = Deutsches Handelsgesetzbuch.

GlU. = Entscheidungen des k. k. Obersten Gerichtshofes, herausgegeben von GLASER und UNGER.

GIUNF. = GLASER-UNGER, neue Folge.

GZ. = Gerichtszeitung.

HHB. = Herrenhausbericht (Bericht der Herrenhauskommission über den Entwurf einer Novelle zum ABGB., Beil. 78 zu den sten. Prot. des Herrenhauses, 21. Session).

JBl. = Juristische Blätter.

OGH. = Oberster Gerichtshof.

OLG. = Oberlandesgericht.

OLGRspr.= Die Rechtsprechung der (deutschen) Oberlandesgerichte, herausgegeben von MUGdaN und FalkmanN.

PrEntw. = Entwurf eines Handelsgesetzbuches für die preußischen Staaten.

Prot. $\quad=$ Protokolle der Kommission zur Beratung eines allgem. deutschen HGB., herausgegeben von LuTz.

R. = Reichsgesetzblatt.

RG. = Entscheidungen des deutschen Reichsgerichtes in Zivilsachen.

ROHG. = Entscheidungen des Reichsoberhandelsgerichtes.

Rspr. = „Rechtsprechung“ (Beilage zu den Mitteilungen des Verbandes österr. Banken und Bankiers).

SchwOR. = Schweizerisches Obligationenrecht vom 30. März 1911.

SZ. $\quad=$ Entscheidungen des österr. Obersten Gerichtshofes in Zivilund Justizverwaltungssachen.

ZBl. = Zentralblatt für die juristische Praxis.

ZHR. = Zeitschrift für Handelsrecht.

Bloß mit dem Namen des Verfassers (Herausgebers) sind zitiert:

DÜRINGER-HAChenburg $=$ Das Handelsgesetzbuch vom 10. Mai 1897, 3. Aufl., unter Leitung von Max Hachenburg, erläutert von BiNG, Breit, Flechtheim, Geiler, Hoeniger, Lehmann, Werner.

Ehren zweig = System des österr. allgem. Privatrechtes von Armin Ehren ZWEIG. 
GoLDSCHMIDT = Handbuch des Handelsrechtes, von GoLdschmidt, 2. Aufl. 1874.

HAHN $=$ Kommentar zum allgem. deutschen Handelsgesetzbuch von Fr. voN Hahn. I. Band (3. Aufl.) 1877; II. Band (2. Aufl.) 1874.

KLANG $=$ Kommentar zum allgem. bürgerl. Gesetzbuche, herausgegeben von KLANG.

OFNER = Der Urentwurf und die Beratungsprotokolle zum ABGB., herausgegeben von OFNer.

STAUB $^{5}=$ Kommentar zum allgem. deutschen Handelsgesetzbuch von Staub, 5. Auflage.

TuHR, BürgR. = Der allgemeine Teil des deutschen bürgerlichen Rechtes, von A. VON TuHR.

TuHR, SchwOR. = Allgemeiner Teil des Schweizerischen Obligationenrechtes, von A. vON TuHr.

ZEILLER = Kommentar über das allgemeine bürgerliche Gesetzbuch von ZEILLER. 